\title{
Dependencia emocional en las relaciones de pareja como Síndrome de Artemisa: modelo explicativo
}

\author{
Emotional dependence on relationships and Artemis syndrome: explanatory model
}

\author{
María de la Villa Moral \\ Universidad de Oviedo, España \\ Carlos Sirvent \\ Fundación Instituto Spiral, Madrid y Oviedo, España. \\ Anastasio Ovejero \\ Universidad de Valladolid, España \\ Glenda Cuetos \\ Universidad de Oviedo, España
}

Rec (31 de marzo de 2017) Acept (22 de septiembre de 2018)

\begin{abstract}
Resumen
La Dependencia Emocional se describe como un tipo de dependencia sentimental caracterizada por la manifestación de interdependencia relacional. Se propone como objetivo establecer un análisis comparativo de su perfil clínico y psicosocial. Para ello, se han seleccionado 880 participantes, de los cuales 277 están filiados como muestra clínica, 311 corresponden a población general y el resto representan muestras de comparación (adictos y familiares no codependientes). Según los resultados hallados, se define el perfil del dependiente emocional como propio de sujetos que experimentan desajustes afectivos en forma de sentimientos negativos (soledad, tristeza, desánimo, culpa, etc.) y vacío emocional junto a deseos de autodestrucción e inescapabilidad emocional. Presentan una dependencia pura (manera de depender de una persona de forma subordinada), con un anhelo irresistible de estar con la persona de la que se depende. Se abunda en la necesidad de implementar propuestas de gestión emocional y de promoción de la salud relacional.

Palabras clave: Dependencia emocional; amor; relaciones de pareja; perspectiva de género.
\end{abstract}

\begin{abstract}
Emotional dependence is described as a type of sentimental dependency characterized by the manifestation of relational interdependence. Its objective is to establish a comparative analysis of its clinical and psychosocial profile. For this purpose, 880 participants were selected, of whom 277 are classified as clinical samples, 311 correspond to the general population and the rest represent comparison samples (addicts and noncodependent relatives). According to the results found, the profile of the emotional dependent is defined as belonging to subjects who experience affective imbalances in the form of negative feelings (loneliness, sadness, discouragement, guilt, etc.) and emotional emptiness together with desires for self-destruction and emotional inescapability. They present a pure dependence (way of depending on a person in a subordinate way), with an irresistible desire to be with the person on whom one depends. It is necessary to implement proposals for emotional management and promotion of relational health.

Keywords: Emotional dependency; love; relationships; gender perspective.
\end{abstract}

Correspondencia: María de la Villa Moral Jiménez, Institución: Universidad de Oviedo, Departamento de Psicología. Dirección Postal: Facultad de Psicología, Plaza de Feijóo, s/n - despacho 211, 33003 Oviedo (España), teléfono: (98) 5103282, fax: (98) 5104144 - (98) 104141, e-mail:mvilla@uniovi.es 


\section{Introducción}

En las sociedades contemporáneas la vida relacional y socioafectiva está sometida a renovadas condiciones psicosociales en las que los conflictos emocionales en las relaciones de pareja e interpersonales son descritos como propios de una suerte de psicopatología de la vida cotidiana. Se apela a los cambios sustanciales que se van produciendo en la manifestación de las emociones y, entre ellas, específicamente en el caso del amor, ya que abundan desregulaciones varias que afectan a la expresión de las emociones y de los sentimientos (Greenberg \& Goldman, 2008; Nussbaum, 2008) y a la propia esfera identitaria (Moral, 2005; Sirvent \& Moral, 2018) asociadas a otros cambios globales (Berscheid, 2010). A nivel investigador, a pesar de que el estudio del amor ha sido considerado como una temática menor dentro de disciplinas como la Psicología Social (Sangrador, 1993), en las últimas décadas se ha tendido a una normalización cientifica, en los términos expresados por García y Cedillo (2011) y, a otro nivel, a una tendencia a la globalización de las emociones y al empoderamiento emocional (Calvo, 2017; Castro, 2012; Santiago, 2018).

El amor es valorado como un sentimiento vital y trayectoria a largo plazo, siendo conceptualizado como una de las emociones más intensas y deseables, según Sternberg (1988). El calificado por Giddens (2000) como amor confluente, por oposición al amor romántico, conlleva una idea de intimidad que contraviene la idea de la entrega incondicional y permanente. Semejante amor líquido (Baumann, 2005) está vinculado al cambio social y a las condiciones postmodernas manifestadas en transformaciones estructurales de la vida social relacionadas con la conducta amorosa, tales como la reorganización de la vida doméstica, los roles de género, las relaciones paterno-filiales y el comportamiento sexual, según el análisis de Crego (2004) (véase Calvo, 2017; Sánchez-Sicilia \& Cubells, 2018). Del comportamiento amoroso se derivan múltiples implicaciones sobre el bienestar físico, psicológico y social y, específicamente, la pareja constituye una fuente de satisfacción psicosocial, estando relacionada la calidad de las relaciones de pareja a los estilos de apego de los individuos (Gómez, Ortiz \& Gómez-López, 2011; Valle \& Moral, 2018). Sin embargo, las nuevas formas de relación amorosa y sus conflictos son una de las señas identificativas del normal caos en el amor, en los términos expresados por Beck \& Beck-Gernsheim (2001). El amor puede convertirse en necesidad y generar conflictos, como en el caso de los dependientes emocionales que desarrollan patrones de vinculación disfuncional. La interrelación puede derivar en apego dependiente con la manifestación de síntomas psicopatológicos de merma de la autonomía personal, necesidad excesiva del otro, síntomas de abstinencia y craving en su ausencia, naturaleza desiderativa de las experiencias y otros signos denotativos (Moral \& Sirvent, 2008, 2009; Sirvent, 2000).

En la dependencia emocional se encuentran implicados aspectos tanto emocionales como cognitivos, motivacionales y comportamentales orientados al otro, así como creencias distorsionadas acerca del amor, de la afiliación e interdependencia y de la vida en pareja, que derivan en insatisfacciones y que se fundamentan sobre demandas afectivas frustradas (Lemos \& Londoño, 2006; Moral \& Sirvent, 2008; Sirvent \& Moral, 2018). También ha sido descrita como un factor explicativo de la violencia intrafamiliar (Moral, García, Cuetos \& Sirvent, 2017). Y es que tanto en las primeras relaciones como en las parejas más consolidadas se manifiestan conductas violentas en mayor o menor medida (Batiza, 2017; Redding, Ruiz, Fernández \& Guijarro, 2017). En estudios como los de Amor, Echeburúa \& Loinaz (2009), en los que se avanza en la clasificación tipológica de los hombres violentos contra su pareja, se incide en las características diferenciales de sus vínculos afectivos, estilos cognitivos y relacionales. En relaciones de pareja con conflictos de violencia física y/o psicológica tiende a afianzarse la idea de que la pareja va cambiar, con lo que se tiende a la justificación de la relación conflictiva (Andrade \& García, 2009; Aráujo, 2008; Loinaz \& Echeburúa, 2012; López-Zafra \& Rodríguez, 2008; Lucariello \& Fajardo, 2011, 2012; Tasso, Brown, Griffo \& Maxwell, 2012). En este sentido, como distorsiones cognitivas en agresores de pareja destaca la negación del problema y los mecanismos de culpabilización de la víctima (Loinaz, 2014), más prevalentes que otras diferencias en variables emocionales (estilo de apego, empatía y autoestima); (Loinaz, Echeburúa, \& Ullate, 2012). Todo ello se fundamenta en mecanismos de desigualdad emocional, en opinión de Verdú (2013), factores clave de desregulaciones a nivel afectivo, con mecanismos de sometimiento y/o sojuzgamiento, juegos de poder y otros signos descriptores, en casos de dependencia emocional. En la base de ello se encuentra la multicausalidad de la problemática investigada y la vinculación entre dependencia emocional y actitudes sexistas, dada su trascendencia en las manifestaciones de violencia en el noviazgo y de maltrato psicológico en particular (Bringas et al., 2017; García et al., 2018; Momeñe, Jáuregui \& Estévez, 2017; Valle \& Moral, 2018). Otro fenómeno observado referente a la dependencia y las relaciones de maltrato es la disociación entre la victimización de violencia por parte de la pareja y la auto-percepción de víctima (Rodríguez-Franco, Antuña-Bellerín, López-Cepero, Rodríguez-Díaz \& Bringas, 2012) siendo, además, difícil establecer la prevalencia (véase Rubio-Garay, López-González, Carrasco \& Amor, 2017).

Destacan en la literatura sobre el tema los estudios sobre los mitos románticos y el concepto de amor en entornos socioculturales como España (véase Barrón, Martínez-Iñigo, 
De Paul \& Yela, 1999; Ferrer, Bosch \& Navarro, 2010). En contextos latinoamericanos también abundan los estudios sobre los factores relacionados con el comportamiento amoroso e implicados en la elección y mantenimiento o ruptura de relaciones íntimas (García-Arango, 2012; Valdez, González, Arce \& López, 2007) o sobre el estudio del amor y la violencia como construcción social (Conde \& Machado, 2011). A nivel aplicado se han emprendido investigaciones sobre las relaciones románticas y el apego ansioso en adolescentes latinoamericanos (Lemos \& Londoño, 2006; Penagos, Rodríguez, Carrillo \& Castro, 2006), así como estudios sobre la regulación de los vínculos románticos en jóvenes (Bautista, 2017; Jaller \& Lemos, 2009; Lemos, Jaller, González, Díaz \& De la Ossa, 2011; Martínez \& Ripo11-Núñez, 2010) o el estudio de la violencia en sus relaciones de noviazgo (Blázquez, Moreno \& García-Baamonde, 2009; Póo \& Vizcarra, 2008; Rey-Anacona, 2013; Rey-Anacona, Mateus-Cubides \& Bayona-Arévalo, 2010; Redondo, Inglés \& García, 2017; Soriano, 2011; Vizcarra \& Póo, 2011), por citar solo algunos ejemplos. Se describen afectos y apegos idealizados, interpretados como positivos y necesarios, que no solo dañan a los dependientes emocionales, sino que también afectan a sus relaciones intrafamiliares (véase $\mathrm{Hu}$ prich, Rosen \& Kiss, 2013), de ahí la importancia de evaluar tales impactos y sus consecuencias clínicas y psicosociales.

Fundamentado lo anterior, en este estudio se propone como objetivo profundizar en la delimitación conceptual de la Dependencia Emocional con una propuesta de modelo explicativo y establecer un estudio comparativo de su perfil respecto a otras Dependencias Sentimentales, tales como la Codependencia (relación de dependencia que establece un familiar respecto a un consumidor de drogas y/o con otra enfermedad crónica) y la Bidependencia (hábito relacional acomodaticio típico de un adicto o ex adicto a drogas), partiendo del análisis de los factores implicados en su definición. Como hipótesis de trabajo se propone que se confirmará la existencia de diferencias significativas en los perfiles sindrómicos entre los dependientes emocionales y las correspondientes muestras clínicas, de comparación (adictos y familiares no codependientes) y población general, indicativos de mayor patología en los dependientes emocionales en descriptores de interdependencia relacional.

\section{Método}

\section{Participantes}

Se han seleccionado diversas muestras clínicas mediante un diagnóstico con acuerdo inter-jueces y procedimiento de doble ciego llevado a cabo por el equipo multidisciplinar de psicoterapeutas de "Fundación Instituto Spiral" (Oviedo y Madrid, España). La muestra total está integrada por 880 sujetos, de los cuales el 11,4\% ( $\mathrm{n}=100)$ están filiados como Dependientes Emocionales (DE), el 5,9\% $(\mathrm{n}=52)$ como Codependientes (CDP) y el $8,5 \%(\mathrm{n}=75)$ como Bidependientes (BDP). Asimismo, han sido seleccionados mediante un muestreo aleatorio simple de la población del Principado de Asturias (España) 311 sujetos de población general que representan el $35,3 \%$ de la muestra total. Como grupos de comparación se han seleccionado 295 adictos en tratamiento en "Fundación Instituto Spiral" y 47 familiares no codependientes, que representan, respectivamente, el $33,5 \%$ y el $5,3 \%$ de la muestra total.

En concreto, han participado en el estudio cien casos de sujetos diagnosticados como Dependientes Emocionales de los cuales el 73,1\% $(n=57)$ son mujeres y el $26.9 \%(n=21)$ restante hombres. De acuerdo a la estratificación por edades, la media se halla en 38.84 años $(D E=9,554)$, situándose la moda en 39 años, el mínimo de edad en 20 y el máximo en 61. Casi la mitad de los mismos están solteros (44,9\%), más de un tercio declara haber tenido dos matrimonios/parejas (33,3\%), una cuarta parte una única pareja estable, un 19,4\% ha convivido con tres parejas y un $16,7 \%$ con cuatro o más. Respecto a otras variables sociodemográficas de interés un $27,0 \%$ de la muestra de dependientes emocionales ejercen profesiones liberales/funcionariado y un $28,4 \%$ son obreros cualificados.

También se han seleccionado diversas subpoblaciones clínicas de pacientes con Bidependencia $(\mathrm{n}=75)$ y Codependencia $(\mathrm{n}=52)$, adictos en tratamiento $(\mathrm{n}=295)$ y familiares no codependientes $(\mathrm{n}=47)$, cuyas características se describen en la Tabla 1.

De los 311 sujetos seleccionados de la población general del Principado de Asturias (España), de acuerdo a la adscripción por género, componen la muestra un 53,1\% de mujeres (46,9\% varones), con edades comprendidas entre los 18 y los 73 años (media $=36,06, D E=12,154$ ), predominantemente solteros $(67,9 \%, \mathrm{n}=209)$, con estudios universitarios cursados (Grado Medio, 19,7\%, n=61; Grado Superior, $25,5 \%, \mathrm{n}=78$ ), de nivel socioeconómico medio $(84,2 \%, n=202)$, de entornos familiares con cuatro o menos miembros $(81,4 \%)$, y que han convivido a lo largo de su vida con una $(40,9 \%, \mathrm{n}=124)$ o dos parejas $(28,1 \%, \mathrm{n}=85)$. 
Tabla 1. Variables sociodemográficas por tipo de subpoblación clínica, general y de comparación $(\mathrm{N}=880)$.

\begin{tabular}{|c|c|c|c|c|c|c|}
\hline $\begin{array}{l}\text { Datos } \\
\text { sociodemográficos }\end{array}$ & $\begin{array}{l}\text { DE } \\
\% \mathrm{n}\end{array}$ & $\begin{array}{l}\text { BDP } \\
\% \mathrm{n}\end{array}$ & $\begin{array}{l}\text { CDP } \\
\% \mathrm{n}\end{array}$ & $\begin{array}{l}\text { G. Control } \\
\% \mathrm{n}\end{array}$ & $\begin{array}{l}\text { Adictos } \\
\% \mathrm{n}\end{array}$ & $\begin{array}{l}\text { Familiares no CDP } \\
\% \mathrm{n}\end{array}$ \\
\hline Género & $\begin{array}{l}\text { Femenino } \\
73,1(57)\end{array}$ & $\begin{array}{l}\text { Masculino } \\
55,6(35)\end{array}$ & $\begin{array}{l}\text { Femenino } \\
79,5(31)\end{array}$ & $\begin{array}{l}\text { Femenino } \\
53,1(173)\end{array}$ & $\begin{array}{l}\text { Masculino } \\
64,9(191)\end{array}$ & $\begin{array}{l}\text { Femenino } \\
53,2(25)\end{array}$ \\
\hline \multirow[t]{2}{*}{ Edad } & 20 a 61 & 17 a 55 & 22 a 73 & 17 a 62 & 17 a 65 & 25 a 63 \\
\hline & $\begin{array}{l}\text { X } 38,84 \\
\text { (DT } 9,554)\end{array}$ & $\begin{array}{l}\text { X } 36,87 \\
\text { (DT } 9,722)\end{array}$ & $\begin{array}{l}\text { X 49,40 } \\
\text { (DT } 11,598)\end{array}$ & $\begin{array}{l}\text { X } 38,84 \\
\text { (DT } 12,154)\end{array}$ & $\begin{array}{l}\text { X } 37,75 \\
\text { (DT } 9,554)\end{array}$ & $\begin{array}{l}\text { X } 47,91 \\
\text { (DT 10,92) }\end{array}$ \\
\hline Estado Civil & $\begin{array}{l}\text { Soltero } \\
44,9(35)\end{array}$ & $\begin{array}{l}\text { Soltero } \\
67,6(50)\end{array}$ & $\begin{array}{l}\text { Casado } \\
70,0(36)\end{array}$ & $\begin{array}{l}\text { Soltero } \\
67,9(209)\end{array}$ & $\begin{array}{l}\text { Soltero } \\
59,6(167)\end{array}$ & $\begin{array}{l}\text { Casado } \\
72,2(34)\end{array}$ \\
\hline Profesión & $\begin{array}{l}\text { Funcionario } \\
27(20)\end{array}$ & $\begin{array}{l}\text { Obrero } \\
\text { cualificado } \\
28,6(21)\end{array}$ & $\begin{array}{l}\text { Ama de casa } \\
45,6(23)\end{array}$ & $\begin{array}{l}\text { Obrero } \\
\text { cualificado } \\
19,9(61)\end{array}$ & $\begin{array}{l}\text { Obrero no } \\
\text { cualificado } \\
36,5(107)\end{array}$ & $\begin{array}{l}\text { Ama de casa } \\
32,9(15)\end{array}$ \\
\hline Nivel cultural & $\begin{array}{l}\text { Bachiller } \\
25,3(19)\end{array}$ & $\begin{array}{l}\text { Bachiller } \\
27,0(20)\end{array}$ & $\begin{array}{l}\text { Primarios } \\
24,0(14)\end{array}$ & $\begin{array}{l}\text { Bachiller } \\
27,7(84)\end{array}$ & $\begin{array}{l}\text { Primarios } \\
37,5(110)\end{array}$ & $\begin{array}{l}\text { Primarios } \\
21,0(10)\end{array}$ \\
\hline Nivel socioeconómico & $\begin{array}{l}\text { Medio } \\
72,1(44)\end{array}$ & $\begin{array}{l}\text { Medio } \\
81,8(61)\end{array}$ & $\begin{array}{l}\text { Medio } \\
80,7(41)\end{array}$ & $\begin{array}{l}\text { Medio } \\
84,2(202)\end{array}$ & $\begin{array}{l}\text { Medio } \\
65,2(184)\end{array}$ & $\begin{array}{l}\text { Medio } \\
79,8(37)\end{array}$ \\
\hline $\begin{array}{l}\mathrm{N}^{\circ} \text { de miembros en el } \\
\text { hogar }\end{array}$ & $\begin{array}{l}2 \\
24,4(18)\end{array}$ & $\begin{array}{l}3 \\
35,3(26)\end{array}$ & $\begin{array}{l}4 \\
36,2(18)\end{array}$ & $\begin{array}{l}4 \\
42,3(130)\end{array}$ & $\begin{array}{l}4 \\
27,8(82)\end{array}$ & $\begin{array}{l}4 \\
37,7(18)\end{array}$ \\
\hline $\mathrm{N}^{\mathrm{o}}$ de hijos & $\begin{array}{l}0 \\
41,7(30)\end{array}$ & $\begin{array}{l}0 \\
45,7(34)\end{array}$ & $\begin{array}{l}1 \\
31,3(1)\end{array}$ & $\begin{array}{l}0 \\
55,4(165)\end{array}$ & $\begin{array}{l}0 \\
47,3(139)\end{array}$ & $\begin{array}{l}2 \\
38,7(19)\end{array}$ \\
\hline $\mathrm{N}^{\mathrm{o}}$ de hermanos & $\begin{array}{l}2 \\
36,6(26)\end{array}$ & $\begin{array}{l}3 \\
34,4(25)\end{array}$ & $\begin{array}{l}3 \\
37,2(18)\end{array}$ & $\begin{array}{l}2 \\
40,0(124)\end{array}$ & $\begin{array}{l}3 \\
32,7(96)\end{array}$ & $\begin{array}{l}3 \\
35,2(16)\end{array}$ \\
\hline $\mathrm{N}^{0}$ de parejas & $\begin{array}{l}2 \\
33,3(24)\end{array}$ & $\begin{array}{l}3 \\
30,6(230)\end{array}$ & $\begin{array}{l}1 \\
61,3(37)\end{array}$ & $\begin{array}{l}1 \\
40,9(124)\end{array}$ & $\begin{array}{l}3 \\
22,6(66)\end{array}$ & $\begin{array}{l}1 \\
71,8(34)\end{array}$ \\
\hline
\end{tabular}

Nota $. \mathrm{DE}=$ Dependencia Emocional, $\mathrm{BDP}=$ Bidependencia y $\mathrm{CDP}=$ Codependencia.

\section{Instrumentos de evaluación}

En este estudio se ha empleado el Inventario de Relaciones Interpersonales y Dependencias sentimentales I.R.I.D.S.-100 (anterior T.D.S.-100) de Sirvent \& Moral (2005) compuesto por 100 ítems evaluados mediante escala Likert de cinco puntos (Muy de Acuerdo a Muy en Desacuerdo). Según la estructura factorial obtenida por rotación varimax está integrado por 7 subescalas (Interdependencia, Acomodación situacional, Autoengaño, Sufrimiento, Identidad y límites relacionales, Antecedentes personales y Heterocontrol) y 23 factores sindrómicos. Dados los objetivos planteados, se ha priorizado el análisis de la Escala de Interdependencia y de los factores pertinentes al objeto de estudio, que, tal como se ha indicado, valora el apego dependiente en la interrelación para lo cual se apoya en tres factores: la necesidad excesiva del otro, los síntomas de abstinencia y craving en su ausencia y la naturaleza desiderativa de los estímulos y experiencias junto al otro/a. El inventario
I.R.I.D.S. -100 es fiable (Alfa de Cronbach $=.976$ para una muestra total de 880 sujetos con valores que oscilan entre .878 para la Subescala de Sentimientos Negativos y .847 para la de Acomodación Situacional), válido, consistente, y diferencia cuatro tipos de dependencia interpersonal: Relacional, Emocional, Bidependencia y Codependencia (Sirvent \& Moral, 2018).

Como cuestionarios de anclaje se han empleado el Inventario de Dependencias Interpersonales (I.D.I.) de Hirschfeld, Klerman, Gough, Barret, Korchin y Chodoff (1997); el Co-dependency Assessment Tool (CODAT) adaptado por Pérez y Delgado (2003) y la Escala de Bidependencia (BDP-25) de Sirvent \& Martínez (2001).

\section{Procedimiento y análisis de datos}

Dado nuestro interés investigador en la temática se estableció un acuerdo de colaboración entre dos Unidades de Investigación, una académica y otra clínica. En la fase 
inicial se ha realizado un riguroso diagnóstico diferencial de las dependencias sentimentales respecto a otras patologías (apego ansioso, trastorno de personalidad límite, sociotropía, etc.) emprendido por un equipo multidisciplinar, a través del procedimiento de doble ciego y mediante acuerdo inter-jueces. Asimismo, se han obtenido los informes favorables de las Comisiones éticas y se han cumplido las directrices de confidencialidad. La recogida de la información se ha llevado a cabo por profesionales dentro del programa terapéutico contando con suficientes garantías de anonimato de la muestra, tales como la asignación de un código identificativo, dado el carácter longitudinal de la investigación. El procesamiento y tratamiento estadístico de los datos se ha llevado a cabo mediante el programa SPSS versión 19.0 y se han efectuado análisis descriptivos y de comparación de medias (ANOVAs), así como técnicas estadísticas de análisis multivariado para la exploración de las relaciones entre las variables.

\section{Resultados}

De acuerdo con los resultados hallados, se define el perfil del dependiente emocional según criterios diferenciales que exploran craving, abstinencia, búsqueda de sensaciones, control y dominio y autoengaño, entre otros indicadores. Se ofrece un análisis de comparación de medias tanto de las siete dimensiones que integran el IRIDS-100 (véase Tabla 2) desglosado por tipo de dependencia (Dependencia Emocional, Bidependencia y Codependencia), así como en población general y en las submuestras de comparación (adictos y familiares no codependientes). Para interpretar los resultados hallados ha de tenerse en cuenta que puntuaciones más bajas son indicativas de mayor patología.

Según el perfil diferencial hallado en los dependientes emocionales se comprueba que:

a) Experimentan desajustes afectivos en forma de Sentimientos negativos (soledad, tristeza, abatimiento, desánimo, culpa, etc.) y un fuerte Vacío emocional junto a deseos de Autodestrucción y un acusado sentimiento de Inescapabilidad emocional, descrita como la sensación de que está atado a la relación de modo que no puede escapar de ella;

b) Presentan una Dependencia pura (manera de depender de una persona de forma subordinada), con un acusado anhelo irresistible de estar con la persona de la que de depende (factor Craving) y la necesidad compulsiva de estar con él/ella experimentando reacciones negativas en su ausencia (factor Abstinencia), en menor medida es un buscador de sensaciones (Dimensión Interdependencia);

c) Sufren vacío emocional y sentimiento crónico de insatisfacción, así como miedo a la soledad y baja tolerancia a la frustración y el aburrimiento (Dimensión Sentimientos Negativos);

d) Tienden al desarrollo de un deseo de focalización en el otro y autonegligencia de modo que centran su atención personal en el sujeto del que dependen incluso descuidando sus propias necesidades (factor Focalización en el otro y autonegligencia);

e) La esfera identitaria también se ve afectada, de manera que se caracterizaría por un Yo débil y por conflictos de Identidad (Dimensión Caracterosis);

$f$ ) Son personas tendentes a acomodarse en las relaciones dejando que sean otros los que decidan por ellos (Dimensión Acomodación);

g) Desarrollan una escasa o nula conciencia de problema, de modo que presentan una incapacidad para darse cuenta de los efectos adversos de la relación, aunque todo el mundo ve el problema menos el afectado (factor Autoengaño), se quejan de que no adoptan soluciones o quiere que éstas vengan de fuera, presentan una escasa o distorsionada conciencia de problema y tienden a cometer los mismos fallos con los sujetos de los que dependen u otras personas no aprendiendo de los errores (factor Reiteración) (Dimensión Autoengaño) y, entre otras señas distintivas de interés

h) Presentan antecedentes familiares de historial psicopatológico y perciben carencias afectivas en la infancia (véase Tabla 2).

Tabla 2. Comparaciones de medias (ANOVA) y comparaciones Post-hoc entre las subpoblaciones clínicas, de comparación y control en las Escalas del I.R.I.D.S.-100.

\begin{tabular}{lll}
\hline Escalas del I.R.I.D.S.-100 & F & Comparaciones Post-hoc (Tukey-b) \\
\hline Interdependencia & $49,940^{*}$ & DE, BDP $<$ CDP, Adictos, G.Control $<$ Familiares no-CDP \\
Acomodación Situacional & $40,971^{*}$ & DE $<$ BDP, CDP, G.Control $<$ Adictos, Familiares no-CDP \\
Autoengaño & $51,025^{*}$ & BDP, DE $<$ CDP $<$ Adictos $<$ G.Control $<$ Familiares no-CDP \\
Sentimientos Negativos & $45,199^{*}$ & DE $<$ BDP, CDP, Adictos $<$ G.Control $<$ Familiares no-CDP \\
Caracterosis & $50,016^{*}$ & DE, CDP, BDP $<$ Adictos $<$ G.Control $<$ Familiares no-CDP \\
Antecedentes personales & $35,656^{*}$ & BDP, D.E., Adictos $<$ CDP $<$ G.Control $<$ Familiares no-CDP \\
Triada Codependiente / & $31,825^{*}$ & CDP $<$ DE, BDP $<$ Adictos $<$ G.Control $<$ Familiares no-CDP \\
Heterocontrol & & \\
\hline
\end{tabular}

Nota. ${ }^{*} \mathrm{p}<, 0001$ D.E. $=$ Dependientes Emocionales, $\mathrm{BDP}=$ Bidependendientes, $\mathrm{CDP}=$ Codependientes. $\mathrm{GC}=$ Grupo Control, $\mathrm{AD}=\mathrm{Adictos}, \mathrm{FAM}=\mathrm{Familiares}$ no CDP 
Se ha realizado un Análisis de Varianza Multivariado para controlar la posible acción moduladora de variables asociadas a tales resultados como son las diferencias intergénero. Para controlar tal efecto de covariación se ha propuesto la realización de un contraste multivariado con variable codependiente Género por tipo de dependencia, resultando la intersección significativa en el caso de los dependientes emocionales (Traza de Hotelling F=63,673), codependientes (Traza de Hotelling $\mathrm{F}=29,820$ ) y bidependientes (Traza de Hotelling $\mathrm{F}=69,357$ ). Asimismo, se ha efectuado un contraste multivariado incluyendo las variables psicosociales, sociodemográficas y clínicas de interés, manteniendo controlada la influencia del género (véase Tabla 3).

Tabla 3. Contrastes multivariados MANOVA. Efectos inter-sujetos por tipo de Dependencia Sentimental (Dependencia Emocional, Bidependencia y Codependencia).

\begin{tabular}{lccc}
\hline Modelo corregido & $\mathrm{F}$ & $\mathrm{F}$ & $\mathrm{F}$ \\
Variables dependientes & $\mathrm{DE}$ & $\mathrm{BDP}$ & $\mathrm{CDP}$ \\
\hline Diagnóstico actual: Problemas Psicológicos &, 810 &, 773 &, 775 \\
Diagnóstico actual: Problemas Adictivos & $1,735^{*}$ & $2,503^{*}$ &, 823 \\
Número de parejas &, 869 & 1,030 &, 710 \\
Historial: Problemas Psicológicos &, 694 & 1,692 &, 777 \\
Historial: Problemas Adictivos & $1,696^{* *}$ & $1,671^{* *}$ & 1,541 \\
Antecedentes familiares paternos &, 435 &, 368 &, 574 \\
Antecedentes familiares materno &, 869 & $2,241^{*}$ &, 882 \\
¿Tienes/has tenido conflictos relevantes o importantes en tu relación de pareja? &, 826 & 1,183 &, 617 \\
¿Tienes/has tenido conflictos relevantes o importantes en tus relaciones & 1,093 & 1,135 &, 996 \\
familiares? & & & \\
Heterocontrol & $2,366^{*}$ & $5,274^{*}$ & $1,737^{* *}$ \\
Acomodación & $2,050^{* *}$ &, 861 & $2,093^{*}$ \\
Autoengaño & $1,650^{* *}$ & $1,962^{* *}$ & $2,231^{*}$ \\
Caracterosis & $3,361^{*}$ & 1,240 & $4,030^{*}$ \\
Antecedentes personales &, 781 & $1,626^{* *}$ &, 599 \\
Sentimientos Negativos & $3,453^{*}$ & 1,273 & $3,073^{*}$ \\
Heterocontrol & $2,045^{* *}$ & $1,308^{* *}$ & $5,375^{*}$ \\
\hline
\end{tabular}

DE $\mathrm{R}^{2}=, 813\left(\mathrm{R}^{2}\right.$ corregida $\left.=, 578\right) \mathbf{B D P}^{2}=, 853\left(\mathrm{R}^{2}\right.$ corregida $\left.=, 692\right) \mathrm{CDP} \mathrm{R}^{2}=, 847\left(\mathrm{R}^{2}\right.$ corregida $\left.=, 690\right)$

Nota. $* \mathrm{p}<, 001 * * \mathrm{p}<, 05$

D.E. $=$ Dependientes Emocionales, $\mathrm{BDP}=$ Bidependendientes, $\mathrm{CDP}=$ Codependientes

A objeto de determinar la adecuación de los valores pronosticados con los observados en el constructo objeto de análisis se ha realizado un análisis de regresión logística, empleándose como covariables las dimensiones analizadas. Se ha utilizado el procedimiento adelante condicional y se han analizado los residuos logit, incidiéndose en el grado de adecuación o no de las covariables seleccionadas y en las probabilidades de los valores pronosticados, así como en la importancia relativa de cada cual y su valor explicativo (véase Tablas 4 y 5). La correlación múltiple entre el criterio y las variables independientes seleccionadas es elevada $(\mathrm{R}=.936, \mathrm{R} 2=.877)$, de modo que las variables seleccionadas explican cerca del ochenta y ocho por ciento $(87,6 \%)$ de la varianza criterio. Se constata que las variables predictoras que más explican la variable criterio son las correspondientes a los Sentimientos Negativos $(\beta=.893 ; \mathrm{p}<.000)$, así como los problemas de identidad (Caracterosis, $\beta=.318, \mathrm{p}<.000$ ) con el desarrollo de pseudosimbiosis (no sentirse completo/a sin el otro), el proceso gradual de Interdependencia $(\beta=.318$, $\mathrm{p}<.000)$ e influyen los Antecedentes familiares $(\beta=.251$, $\mathrm{p}<.000)$ y manifestaciones de Heterocontrol Relacional $(\beta=.083, \mathrm{p}<.05)$. 
Tabla 4. Resumen de la Regresión Logística constructo Dependencia Emocional.

\begin{tabular}{lllllll}
\hline \multicolumn{7}{c}{ Variables explicativas constructo Dependencia Emocional } \\
\hline Modelo & $\mathrm{R}$ & $\mathrm{R} 2$ & $\mathrm{R} 2$ corregida & Error típ. de la estimación & Sig. del cambio en F & Cambio en R2 \\
\hline 1 &, $893(\mathrm{a})$ &, 797 &, 796 &, 28811 &, 797 & 632,439 \\
2 &, $920(\mathrm{~b})$ &, 846 &, 844 &, 25149 &, 049 & 51,300 \\
3 &, $932(\mathrm{c})$ &, 869 &, 866 &, 23298 &, 023 & 27,432 \\
4 &, $935(\mathrm{~d})$ &, 874 &, 871 &, 22942 &, 005 & 5,973 \\
5 &, $936(\mathrm{e})$ &, 877 &, 873 &, 22715 &, 003 & 4,168 \\
\hline
\end{tabular}

a Variables predictoras: (Constante), Dimensión Sentimientos Negativos

b Variables predictoras: (Constante), Dimensión Sentimientos Negativos, Caracterosis

c Variables predictoras: (Constante), Dimensión Sentimientos Negativos, Caracterosis, Interdependencia

d Variables predictoras: (Constante), Dimensión Sentimientos Negativos, Caracterosis, Interdependencia, Antecedentes Personales

e Variables predictoras: (Constante), Dimensión Sentimientos Negativos, Caracterosis, Interdependencia, Antecedentes personales, Heterocontrol.

La matriz de coeficientes del modelo de regresión logística Dependencia Emocional se presenta en la Tabla 5.

Se confirma que algunas de las características descriptoras más destacadas hacen referencia a: $a$ ) la posesividad y el desgaste energético psicofísico intenso, b) la incapacidad para romper ataduras (Inescapabilidad emocional), c) la experimentación de desajustes afectivos en forma de Sentimientos Negativos (culpa, vacío emocional, miedo al abandono), d) los desajustes identitarios (Caracterosis) con pseudosimbiosis (no estar completo sin el otro) y de un Ego débil a modo de frontera interpersonal permeable, e) el desarrollo de un locus de control externo, $f$ ) la influencia de los antecedentes familiares de desapego afectivo en la infancia, $g$ ) la elaboración de una escasa o parcial comprensión del problema con mecanismos de Autoengaño, $\mathrm{y}$, asimismo, h) la tendencia a orientarse a la otra personal (Heterocontrol) con Focalización el otro con autodescuido y autonegligencia y Orientación rescatadora con sobreprotección, pseudoaltruismo y abnegación. Un regulador crítico es el Autoengaño en el que incurren los dependientes emocionales de forma consciente, dejándose llevar por las distorsiones de los efectos adversos de la relación, incurriendo en errores similares con sus sucesivas parejas (Reiteración) y empleando mecanismos de negación y no afrontamiento que les permiten instalarse progresiva e insidiosamente en una recreación idealizada de su relación que, al deteriorarse, van adaptando cognitivamente a sus creencias y anhelos.

Tabla 5. Coeficientes del Modelo de Regresión Logística del constructo Dependencia Emocional.

\begin{tabular}{llccccc}
\hline \multicolumn{2}{l}{$\begin{array}{l}\text { Coeficientes (a) } \\
\text { Modelo }\end{array}$} & \multicolumn{2}{l}{$\begin{array}{l}\text { Coeficientes no } \\
\text { estandarizados }\end{array}$} & $\begin{array}{c}\text { Coeficientes } \\
\text { estandarizados }\end{array}$ & T & Sig. \\
\hline \multicolumn{2}{l}{ (a) variable dependiente: Dependencia Emocional } & B & Error típ. & Beta & B & Error típ. \\
\hline $1 \quad$ (Constante) & 4,34 &, 087 & & 4,973 &, 000 \\
& Sentimientos Negativos & 8,62 &, 034 &, 893 & 25,148 &, 000 \\
& (Constante) &,- 202 &, 117 & & $-1,726$ &, 086 \\
& Sentimientos Negativos &, 641 &, 043 &, 665 & 14,952 &, 000 \\
& Caracteroris &, 401 &, 056 &, 318 & 7,162 &, 000 \\
& (Constante) &,- 249 &, 109 & & $-2,290$ &, 023 \\
& Sentimientos Negativos &, 535 &, 045 &, 554 & 11,974 &, 000 \\
& Caracteroris &, 313 &, 054 &, 249 & 5,757 &, 000 \\
& Interdependencia &, 207 &, 040 &, 225 & 5,238 &, 000 \\
& (Constante) &,- 190 &, 110 & & $-1,730$ &, 086 \\
& Sentimientos Negativos &, 545 &, 044 &, 564 & 12,333 &, 000
\end{tabular}




\begin{tabular}{llccccc}
\hline $\begin{array}{l}\text { Coeficientes (a) } \\
\text { Modelo }\end{array}$ & \multicolumn{2}{c}{$\begin{array}{l}\text { Coeficientes no } \\
\text { estandarizados }\end{array}$} & $\begin{array}{c}\text { Coeficientes } \\
\text { estandarizados }\end{array}$ & T & Sig. \\
\hline (a) variable dependiente: Dependencia Emocional & B & Error típ. & Beta & B & Error típ. \\
\hline \multicolumn{2}{c}{ Caracterosis } &, 319 &, 054 &, 254 & 5,948 &, 000 \\
& Interdependencia &, 231 &, 040 &, 251 & 5,752 &, 000 \\
$\quad$ Antecedentes personales &,- 056 &, 023 &,- 079 & $-2,444$ &, 016 \\
5 &,- 233 &, 111 & & $-2,104$ &, 037 \\
$\quad$ Constante) &, 518 &, 046 &, 537 & 11,358 &, 000 \\
$\quad$ Sentimientos Negativos &, 254 &, 062 &, 202 & 4,110 &, 000 \\
$\quad$ Caracterosis &, 251 &, 041 &, 273 & 6,132 &, 000 \\
$\quad$ Interdependencia &,- 056 &, 022 &,- 079 & $-2,477$ &, 014 \\
$\quad$ Antecedentes personales &, 095 &, 047 &, 083 & 2,042 &, 043 \\
\hline
\end{tabular}

\section{Discusión}

En la actualidad las relaciones de pareja se hallan sometidas a unas singularidades, presiones y simbolismos que las caracterizan, máxime ante unas condiciones relacionales de cambios en los estilos de expresión de los afectos y sentimientos (Freitas-Magalhães, 2007; Greenberg \& Goldman, 2008; Hicks \& Hicks, 2008; Nussbaum, 2008) propias de la globalización de las emociones, en el sentido expresado por Castro (2012), características de este nuevo arte de amar y depender (Chaumier, 2006).

En este estudio se ha comprobado que los dependientes emocionales son personas vulnerables emocionalmente que manifiestan una ceguera hacia el otro, lo cual se podría explicar por la conjunción de ilusiones y/o atribuciones, hedonismo y expectativas. De acuerdo con los resultados del perfil diferencial respecto a otras dependencias sentimentales (codependencia y bidependencia), así como respecto a población general y muestras de comparación (adictos y familiares no codependientes) se ha comprobado que la dependencia emocional cursa con sentimientos negativos (soledad, tristeza, abatimiento, desánimo, culpa, etc.), insatisfacción, miedo a la soledad y baja tolerancia a la frustración y el aburrimiento, así como un fuerte vacío emocional junto a deseos de autodestrucción, tienden a experimentar un estado de ánimo medio disfórico y un acusado sentimiento de inescapabilidad emocional. Los dependientes emocionales presentan una manera de depender de una persona de forma subordinada, con un acusado anhelo irresistible de estar con la persona de la que se depende, tendiendo al desarrollo de un deseo de focalización en el otro y autonegligencia, así como conflictos identitarios. Asimismo, son personas tendentes a acomodarse en las relaciones dejando que sean otros los que decidan por ellos y desarrollan una escasa o nula conciencia de problema. La tendencia a orientarse a la otra personal (Heterocontrol) con focalización en la persona de la que depende con autodescuido y autonegligencia constituye otro signo patognomónico. Este perfil va en la dirección de otros estudios con personas con dependencia emocional (Lemos \& Londoño, 2006; Lemos et al., 2011), siendo la disfuncionalidad, saliencia e intensidad de los afectos toxicofílicos elementos claves de esa vinculación emocional a los otros que se convierte en dependencia (véase Moral \& Sirvent, 2008, 2009; Sirvent \& Moral, 2018). De este modo, con la etiqueta de Síndrome de Artemisa puede ser calificada la dependencia emocional al tomar como referencia simbólica el duelo de Artemisa, quien embargada por la enorme tristeza de la pérdida de su esposo mezclaba las cenizas de éste en sus bebidas diarias con lo que experimentaba un placer orgiástico y así se fue apagando gradualmente de duelo hasta su propia muerte. Se significa así el importante peso específico de los sentimientos adversos en la nosología afectivo-dependiente.

Como limitaciones del estudio se plantean las relacionadas con los estudios descriptivos de corte transversal, entre ellas las dificultades de precisar la asociación real entre las variables, así como las derivadas de la selección muestral y en el empleo de medidas autoinformadas (sesgo de deseabilidad social), que se intentaron contrarrestar mediante la garantía del anonimato y la confidencialidad de los datos.

Como líneas prospectivas de investigación en esta temática, basándonos en los resultados hallados, se incide en la importancia de profundizar en el estudio comprehensivo de la psicopatología del amor en las relaciones de pareja (Echeburúa, 2010; Sophia, Tavares \& Zilberman, 2007), en el análisis integral de las emociones y los sentimientos (Angulo, 2012; Retana \& Sánchez, 2010; Manassero, 2013; Zaragoza, 2013), así como en el proceso de construcción social del amor (Belli, Harré \& Iñiguez, 2010; Juan, 2011; Moral, 2005). También incidimos en la necesidad de redimensionar 
los significados atribuidos a los roles femeninos (Charlon, 2009; Valor-Segura, Expósito \& Moya, 2010) y en el desafortunado tránsito del amor romántico a la violencia en las relaciones de pareja (Ferrer \& Bosh, 2013, 2014; Garaigordobil, 2013; Giordano, Soto, Manning \& Longmore, 2010; Horno, 2009; Valle \& Moral, 2018). Específicamente, se ha de adoptar una perspectiva de género en dependencia emocional, dada la necesidad de profundizar en las claves socioculturales de tales patrones relacionales y en sus simbolismos afianzados en el imaginario colectivo (Moral et al., 2017; Moral \& Sirvent, 2008, 2014). En este sentido, se ha de seguir trabajando en políticas de igualdad y de reducción de la discriminación por género, dada la vinculación entre la dependencia emocional y los procesos de violencia interpersonal en las relaciones de pareja (Boira \& Jodrá, 2013; Moral et al., 2017; Sierra, Bermúdez, Buela-Casal, Salinas \& Monge, 2014). Modificar las representaciones estructurales de las creencias amorosas en las representaciones sociales acerca de relaciones de pareja resulta de gran interés (Luiz de Andrado \& Wachelke, 2011), lo cual está influido por su propia cosmovisión romántica, tal y como sugiere Sebold (2011). Asimismo, se ha de abundar en la conveniencia de educar las emociones desde la escuela incidiendo en la implementación de propuestas de gestión emocional y promoción de la salud relacional (Ali et al., 2013; Fossas, 2013; Martín, 2013; Wolfe, Crooks \& Hughes, 2013), promover programas de prevención de la violencia en el noviazgo (Póo \& Vizcarra, 2011; Rodríguez-Franco et al., 2017) y profundizar en objetivos preventivos e intervención integral en dependencias sentimentales (Moral \& Sirvent, 2014).

\section{Referencias}

Ali, A., Ambler, G., Strydom, A., Rai, D., Cooper, C., McManus, S., Weich, S., Meltzer, H., Dein, S. \& Hassiotis, A. (2013). The relationship between happiness and intelligent quotient: The contribution of socio-economic and clinical factors. Psychological Medicine, 43, 1303-1312. doi: 1017/S0033291712002139

Amor, P. J., Echeburúa, E. \& Loinaz, I. (2009) ¿Se puede establecer una clasificación tipológica de los hombres violentos contra su pareja? Internacional Journal of Clinical and Health Psychology, 9, 519-539.

Andrade, A. \& García, A. (2009). Atitudes e Crenças sobre o Amor: Versão Brasileira da Escala de Estilos de Amor. Universidade Federal do Espirito Santo. http://interpersonaabpri.files.wordpress.com/2010/12/ atitudes-e-crenc3a7as-sobre-oamor1.

Angulo, K. M. (2012). Emociones y sentimientos: coordenadas históricas y multidisciplinares de un campo de estudio clave. Avances en supervisión educativa: Revista de la Asociación de Inspectores de Educación de España, 16, 1-13.
Barrón, A., Martínez-Iñigo, D., De Paul, P. \& Yela, C. (1999). Romantic beliefs and myths in Spain. The Spanish Journal of Psychology, 2, 64-73. doi: 10.1017/S1138741600005461

Batiza, F. J. (2017). La violencia de pareja: Un enemigo silencioso. Archivos de Criminología, Seguridad Privada y Criminalística, 18, 144-151.

Baumann, Z. (2005). Amor líquido, acerca de la fragilidad de los vínculos humanos. Madrid: FCE.

Bautista, E. (2017). Cortejo y amor entre jóvenes del distrito de Comas (Lima, Perú). Tesis Doctoral: Universidad Complutense de Madrid.

Blázquez, M., Moreno, J. M. \& García-Baamonde, M. E. (2009). Inteligencia emocional como alternativa para la prevención del maltrato psicológico en la pareja. Anales de Psicología, 25(2), 250-260.

Beck, U. \& Beck-Gernsheim (2001). El Normal Caos del Amor. Las nuevas formas de la relación amorosa. Barcelona: Paidós.

Belli, S., Harré, R. \& Iñiguez, L. (2010). Emociones y discurso: una mirada a la narrativa psicológica sobre la construcción social del amor. Prisma Social: revosta de investigación social, 4, 1-45.

Berscheid, E. (2010). Love in the fourth dimension. Annual Review of Psychology, 61, 1-25. doi: 10.1146/annurev.psych.093008.100318

Boira, S. \& Jodrá, P. (2013). Tipología de hombres condenados por violencia de género en un contexto de intervención psicológica en la comunidad. Revista Latinoamericana de Psicología, 45, 289-303. doi: 10.14349/rlp

Bringas-Molleda, C., Estrada-Pineda, C., Suárez-Álvarez, J., Torres, A., Rodríguez-Díaz, F. J., García-Cueto, E. \& Rodríguez-Franco, L. (2017). Actitud sexista y trascendente durante el noviazgo entre universitarios latinoamericanos. Revista Iberoamericana de Psicología y Salud, 8(1), 44-55. doi: 10.23923/j. rips.2017.08.005

Calvo, S. (2017). Amor romántico, amor confluente y amor líquido: apuntes teóricos en torno a los sistemas sociales de comunicación afectiva. Eikasia: Revista de Filosofía, 77, 141-151.

Castro, F. (2012). La cultura (desquiciada) en la época de la globalización de las emociones. Revista de Occidente, 378, 69-88.

Charlon, A. (2009). Cambios y permanencias del rol femenino en las relaciones de pareja. Foro hispánico: revista hispánica de Flandes y Holanda, 34, 45-63.

Chaumier, S. (2006). El nuevo arte de amar. Madrid: Alianza Editorial.

Conde, A. R., \& Machado, C. (2011). Amor e violência na imtimadade: Da essência à construção social. Psicologia \& Sociedade, 23, 494-505.

Crego, A. (2004). Cambio social y comportamiento amoroso: la incertidumbre en el amor postmoderno. Miscelánea: Revista de Ciencias Humanas y Sociales, 62, 13-47.

Echeburúa, E. (2010). La patología del amor en la relación de pareja. Crítica, 966, 39-43.

Ferrer, V. A. \& Bosch, E. (2013). Del amor romántico a la violencia de género: para una coeducación emocional en la agenda educativa. Profesorado: Revista de curriculum y formación del profesorado, 17, 105-122.

Ferrer, V. A. \& Bosch, E. (2014). The measure of the masculinity-feminity construct today: some reflections on the case of the Bem Sex Role Inventory. Revista de psicología Social, 29, 180-207. doi: 10.1080/02134748.2013.878569.

Ferrer, V. A., Bosch, E. \& Navarro, C. (2010). Los mitos románticos en 
España. Boletín de Psicología, 99, 7-31.

Fossas, F. J. (2013). Salud psicológica: Emociones, función y gestión. Integral: Vive mejor en un mundo mejor, 399, 60-65.

Freitas-Magalhães, A. (2007). The Psychology of Emotions: The allure of human face. Oporto: University Fernando Pessoa Press.

Garaigordobil, M. (2013). Sexismo y apego inseguro en la relación de pareja. Revista Mexicana de Psicología, 30, 53-60. doi: 10.5209/ rev_SJOP.2012.v15.n2.38870

García-Arango, G. (2012). El derecho a estar solo, a la pareja, a la familia y al amor en Colombia y algunos países de Latinoamérica. Revista Facultad de Derecho y Ciencias Políticas, 116, 23-50.

García, A. \& Cedillo, R. P. (2011). La normalización científica del amor. A propósito de la perspectiva evolutiva en Psicología. Revista Latinoamericana de Estudios sobre Cuerpos, Emociones y Sociedad (RELACES), 3, 83-95.

García, V., Lana, A., Fernández, A., Bringas, C., Rodríguez-Franco, L. \& Rodríguez-Díaz, F. J. (2018). Actitudes sexistas y reconocimiento del maltrato en parejas jóvenes. Atención Primaria, 50(7), 398-405. doi: 10.1016/j.aprim.2017.04.001

Giddens, A. (2000). La transformación de la intimidad. Sexualidad, amor y erotismo en las sociedades modernas. Madrid: Cátedra.

Giordano, P. C., Soto, D. A., Manning, W. D. \& Longmore, M. L. (2010). The characteristics of romantic relationships associated with teen dating violence. Social Science Research, 39 , 863-874. doi: 10.1016/j. ssresearch.2010.03.009

Gómez, J., Ortiz, M. J. \& Gómez-López, J. (2011). Experiencia sexual, estilos de apego y tipos de cuidados en las relaciones de pareja. Anales de Psicología, 27, 447-456. doi:10.6018/123081

Greenberg, L. S. \& Goldman, R. N. (2008). Emotion-Focused couples therapy: the dynamics of Emotion, Love, and Power. Washington, D.C.: American Psychological Association.

Hicks, E. \& Hiscks, J. (2008). El increíble poder de las emociones: atrévete a dejarte guiar por los sentimientos. Barcelona: Urano.

Hirschfeld, R.A., Klerman, G.L., Gough, H.G.,Barrett, J., Korchin, S.J.\& Chodoff, P.A. (1977). A Measure of interpersonal dependency. Journal of Personality Assessment, 41, 610-618.

Huprich, S., Rosen, A. \& Kiss, A. (2013). Manifestations of interpersonal dependency and depressive subtypes in outpatient psychotherapy patients. Personality and Mental Health, 7, 223-232. doi: 10.1002/ pmh. 1222

Jaller, C. \& Lemos, M. A. (2009). Esquemas desadaptativos tempranos en estudiantes universitarios con dependencia emocional. Acta Colombiana de Psicología, 12, 77-83. http://www.redalyc.org/ pdf/798/79815640008.pdf

Juan, M. L. (2011). El amor romántico es una construcción cultural y social. Agathos: Atención sociosanitaria y bienestar, 11, 58-61.

Lemos, M. \& Londoño, N. H. (2006). Construcción y validación del Cuestionario de Dependencia Emocional en población colombiana. Acta Colombiana de Psicología, 9, 127-140.

Lemos, M., Jaller, C., González, A. M., Díaz, Z. T. \& De la Ossa, D. (2011). Perfil cognitivo de la dependencia emocional en estudiantes universitarios de Medellín. Universitas Psychologica, 11, 395-404. doi: 10.1016/S0034-7450(14)60125-6

Loinaz, I. (2014). Distorsiones cognitivas en agresores de pareja: análisis de una herramienta de evaluación. Terapia Psicológica, 32, 5-17. doi: 10.4067/S0718-48082014000100001

Loinaz, I. \& Echeburúa, E. (2012). Apego adulto en agresores de pareja. Acción Psicológica, 9, 33-46. doi: 10.5944/ap.9.1.435.

Loinaz, I., Echeburúa, E. \& Ullate, M. (2012). Estilo de apego, empatía y autoestima en agresores de pareja. Terapia Psicológica, 30, 61-70. doi: $10.4067 /$ S0718-48082012000200006

López-Zafra, E. \& Rodríguez, N. (2008). Relación entre cultura del honor, celos y satisfacción en la pareja. Boletín de Psicología, 84, 7-22.

Lucariello, E. \& Fajardo, I. (2011). Prevención de la Violencia de Género en los Adolescentes. International Journal of developmental and Educational psychology. Desafios y Perspectivas Actuales de la Psicologia, XXIII, 1, 113-121.

Lucariello, E. \& Fajardo, I. (2012). Estilos de Amor en Mujeres Maltratadas. Journal of developmental and Educational psychology. Familia y Educación en un Mundo en Cambio, XXIV, 1, 345-360.

Luiz de Andrado, A. \& Wachelke, J. (2011). La asociación de configuraciones estructurales de las relaciones románticas con las creencias sobre las relaciones de pareja: un estudio de las representaciones sociales. Anales de Psicología, 27, 834-842. doi:10.6018/135581.

Manassero, M. A. (2013). Emociones: del olvido a la centralidad en la explicación del comportamiento. Las emociones en la enseñanza y el aprendizaje de las ciencias y las matemáticas, 1, 3-18.

Martín, J. (2013). ¿Se pueden educar las emociones desde la escuela? Diálogo: Familia Colegio, 297, 36-44.

Martínez, K. \& Ripoll-Núñez, K. (2010). Consistencia ideal-percepción y regulación en las relaciones románticas de adultos jóvenes: El papel de las variables personales y de la relación. Summa Psicológica, 7(1), 63-78.

Momeñe, J., Jáuregui, P. \& Estévez, A. (2017). El papel predictor del abuso psicológico y la regulación emocional en la dependencia emocional. Behavioral Psychology / Psicología Conductual, 25(1), 65-78.

Moral, M. V. (2005). La rebelión de las emociones y los sentimientos: abordaje psicosocial de las dependencias afectivas y la adicción al amor en mujeres maltratadas. Asociación de Ayuda a Víctimas de Agresiones Sexuales y Violencia Doméstica (Eds.), Dependencia emocional: educación y prevención (pp. 51-89). León, España: ADAVAS.

Moral, M. V. \& Sirvent, C. (2008). Dependencias sentimentales o afectivas: etiología, clasificación y evaluación. Revista Española de Drogodependencias, 33, 145-167.

Moral, M. V., \& Sirvent, C. (2009). Dependencia afectiva y género: perfil sintomático diferencial en dependientes afectivos españoles. Interamerican Journal of Psychology, 43, 230-240.

Moral, M. V. \& Sirvent, C. (2014). Objetivos psicoterapéuticos e intervención integral en Dependencias Sentimentales. Revista Española de Drogodependencias, 39, 38-56.

Moral, M. V., García, A., Cuetos, G. \& Sirvent, C. (2017). Violencia en el noviazgo, dependencia emocional y autoestima en adolescentes y jóvenes españoles. Revista Iberoamericana de Psicología y Salud, 8 (2), 96-107. doi: 10.23923/j.rips.2017.08.009

Nussbaum, M. C. (2008). Paisajes del pensamiento: La inteligencia de 
las emociones. Barcelona: Paidós.

Penagos, A., Rodríguez, M., Carrillo, S. \& Castro, J. (2006). Apego, relaciones románticas y autoconcepto en adolescentes bogotanos. Universitas Psychologica, 5, 21-36.

Pérez, A. \& Delgado, D. (2003). La codependencia en familias de consumidores y no consumidores de drogas: estado del arte y construcción de un instrumento. Psicothema, 15, 381-387.

Póo, A. M. \& Vizcarra, M. B. (2008). Violencia de pareja en jóvenes universitarios. Terapia psicológica, 26, 81-88.

Póo, M. \& Vizcarra, M. B. (2011). Diseño, implementación y evaluación de un programa de prevención de la violencia en el noviazgo. Terapia Psicológica, 29, 213-223.

Redding, E. M., Ruiz, M. T., Fernández, J. \& Guijarro, M. (2017). Gender inequality and violence against women in Spain, 2006-2014: Towards a civilizited society. Gaceta Sanitaria, 31, 82-88. doi:10.1016/j.gaceta.2016.07.025

Redondo, J., Inglés, C. J. \& García, K. (2017). Papel que juega la edad en el noviazgo de estudiantes de la Universidad Pontificia Bolivariana de Bucaramanga. Diversitas: Perspectivas en Psicología, 13(1), 41-54.

Retana, B. E. \& Sánchez, R. (2010). Rastreando en el pasado... formas de regular la felicidad, la tristeza, el amor, el enojo y el miedo. Universitas Psychologica, 9, 179-197.

Rey-Anacona, C. A. (2013). Prevalencia y tipos de maltrato en el noviazgo en adolescentes y adultos jóvenes. Terapia Psicológica, 31, 143-154. doi: 10.4067/S0718-48082013000200001

Rey-Anacona, C. A., Mateus-Cubides, A. M. \& Bayona-Arévalo, P. A. (2010). Malos tratos ejercidos por adolescentes durante el noviazgo: Diferencias por sexo. Revista Mexicana de Psicología, 27, 169-181.

Rodríguez-Franco, L., Antuña-Bellerín, M. A., López-Cepero, J., Rodríguez-Díaz, F. J. \& Bringas, C. (2012). Tolerance towards dating violence in Spanish adolescents. Psicothema, 24(2), 236-242.

Rodríguez-Franco, L., Gracia-Morilla, C., Juarros, J., Fernández, A. \& Rodríguez, F. J. (2017). Agresores generalistas y especialistas en violencia de parejas jóvenes y adolescentes: implicaciones en la implementación de los programas de prevención. Acción Psicológica, 14(2), 1-16. doi: 10.5944/ap.14.2.20

Rubio-Garay, M., López-González, M. A., Carrasco, M. A. \& Amor, P. J. (2017). Prevalencia de violencia en el noviazgo: una revisión sistemática. Papeles del Psicólogo/Psychologist Papers, 38(2), 135-147. doi: 10.23923/pap.psicol2017.2831

Sánchez-Sicilia, A. \& Cubells, J. (2018). Amor, posmodernidad y perspectiva de género: entre el amor romántico y el amor líquido. Investigaciones feministas: papeles de estudios de mujeres, feministas y de género, 9(1), 151-171.

Sangrador, J. L. (1993). Algunas consideraciones sobre el amor romántico. Psicothema, 5, 181-196.

Santiago, M. (2018). Empoderamiento emocional. Cambio en la estructura del reconocimiento individual y en las relaciones interpersonales. Tercio Creciente, 13, 79-96.

Sebold, R.P. (2011). La cosmovisión romántica: siete síntomas y cinco metáforas. Castilla. Estudios de Literatura, 2, 311-323.

Sierra, J. C., Bermúdez, M. P., Buela-Casal, G., Salinas, J. M. \& Monge,
F. S. (2014). Variables asociadas a la experiencia de abuso en la pareja y su denuncia en una muestra de mujeres. Universitas Psychologica, 13. doi:10.11144/Javeriana. UPSY13-1.vaea

Sirvent, C. (2000). Dependencias relacionales: codependencia, bidependencia, dependencia afectiva. Paper presented at the I Symposium Nacional sobre Adicción en la Mujer. Madrid, España.

Sirvent, C. \& Martínez, C. (2001). Escala de Bidependencia BDP-25. Oviedo: Fundación Instituto Spiral.

Sirvent, C. \& Moral, M. V. (2005). Test de Dependencias Sentimentales $T D S-100$. Oviedo, España: Fundación Instituto Spiral.

Sirvent, C. \& Moral, M. V. (2018). Construcción y validación del Inventario de Relaciones Interpersonales y Dependencias Sentimentales (IRIDS-100). Health and Addictions, 18(2), 35-47.

Sophia, E., Tavares, H. \& Zilberman, M. (2007). Pathological Love: is it a New Psychiatric Disorder? Revista Brasileña de Psiquiatría, 29, 55-62. doi: 10.1590/S1516-44462006005000003.

Soriano, A. (2011). La violencia en las relaciones de pareja en estudiantes universitarios: propuestas educativas. Pedagogía Social: revista interuniversitaria, 18, 87-97.

Sternberg (1988). El Triángulo del Amor: Intimidad, Pasión y Compromiso. Barcelona: Paidós.

Tasso, A. F., Brown, D., Griffo, R. \& Maxwell, K. S. (2012). The use of the Adult Attachment Scale with domestically violent men. Journal of Family Violence, 27, 731-739. doi: 10.1007/s10896-012-9466-9

Valdez, J., González, L., Arce, V. \& López J. (2007). La elección real e ideal de pareja: Un estudio con parejas establecidas. Revista Interamericana de Psicología, 41, 1-7.

Valle, L. \& Moral, M. V. (2018). Dependencia emocional y estilo de apego adulto en las relaciones de noviazgo en jóvenes españoles. Revista Iberoamericana de Psicología y Salud, 9 (1), 27-41. doi: 10.23923/j. rips.2018.01.013

Valor-Segura, I., Expósito, F. \& Moya, M. (2010). Emociones poderosas y no poderosas ante conflictos de pareja. Diferencias de Género. Psychosocial Internation, 19, 129-134. doi: 10.5093/in2010v19n2a4

Verdú, A. D. (2013). Conflicto en las relaciones de pareja heterosexuales: la desigualdad emocional. Cuestiones de género: de la igualdad y la diferencia, 8, 165-181.

Vizcarra, M. B. \& Póo, A. M. (2011). Violencia de pareja en estudiantes universitarios del sur de Chile. Universitas Psychologica, 10, 89-98.

Zaragoza, J. M. (2013). Historia de las emociones: una corriente historiográfica en expansión. Asclepio: Revista de Historia de la Medicina y de la Ciencia, 65, 1-10. doi: 10.3989/asclepio.2013.12.

Wolfe, D. A., Crooks, C. V., \& Hughes, R. (2013). The Fourth R: A School - Based Adolescent Dating Violence Prevention Program. Psychosocial Intervention, 23, 193-200. doi: 10.5093/in2011v20n2a7. 